\title{
Short term prospective study of cognitive functioning in lead workers
}

\author{
B T Stollery, D E Broadbent, H A Banks, W R Lee
}

\begin{abstract}
In a short term prospective study 70 male lead workers performed a series of cognitive tasks on three occasions during an eight month period. Concurrently with the cognitive testing, the concentrations of blood lead, zinc protoporphyrin (ZPP) and urinary aminolaevulinic acid (ALA) were measured. Indicators of lead absorption were stable during the study and each subject was allocated to either a low (below $20 \mu \mathrm{g} / \mathrm{dl}$ ), medium (21-40 $\mu \mathrm{g} / \mathrm{dl})$, or high (41-80 $\mu \mathrm{g} / \mathrm{dl})$ group on the basis of their average blood lead concentrations. Performance deficits tended to be restricted to the high lead group and, in general, neither practice nor continued exposure during the study altered the magnitude of these deficits. Themaindeficitwas a slowing of sensory motor reaction time, which was seen most clearly when the cognitive demands of the task were low. In the cognitively simple five choice task, blood lead concentration correlated with impaired decision making, response execution, and "lapses in concentration." In the other cognitive tasks the high blood lead group tended also to be slower by a factor of about 1.08 but the dominance of cognitive over sensory motor demands attenuated the exposure-performance correlations. The high lead group also had difficulty in recalling nouns poorly related to the focus of an earlier semantic classification task. This difficulty increased over time and was one of the few findings that correlated with all measures of lead absorption. It is concluded that the primary psychological profile of lead impair-
\end{abstract}

Age and Cognitive Performance Research Centre, University of Manchester, Manchester M13 9PL

B T Stollery

Department of Experimental Psychology, University of Oxford, South Parks Road, Oxford OX1 3UD

D E Broadbent

Department of Occupational Health, University of Manchester, Manchester M13 9PT

H A Banks, W R Lee ment is one of sensory motor slowing coupled with difficulties in remembering incidental information.

Occupational exposure to inorganic lead has frequently been found to disrupt performance on psychological tasks ${ }^{1-6}$ and the diversity of tasks showing impairment has given rise to the impression that the deficits are correspondingly diverse. It has been pointed out elsewhere ${ }^{6-8}$ that performance requires the coordination of many functionally distinct psychological processes and that performance based attempts to identify and model cognitive disorders due to toxic insult are rarely attempted. It remains to be determined, therefore, whether the diversity of lead induced performance deficits arises from a generalised impairment of cognition, the presence of multiple but functionally distinct impairments, or the disruption of a small set of functions central to many cognitive activities. Therefore, a strong research need exists to develop a more rational and theoretically focused approach to neurotoxic dysfunction than is currently available, preferably one within which some degree of differential diagnosis for the various classes of neurotoxins can be achieved. ${ }^{8}$ In our earlier cross sectional study of lead exposure, cognitive performance was assessed by tasks possessing sufficient internal structure to support functional differentiation. ${ }^{6}$ Although lead disrupted the performance of most tasks, the impairment profile showed a dominant influence on sensory motor functions and only a mild influence on other areas of cognition. These results together with those of other studies ${ }^{5}$ weaken the notion of global cognitive impairments and strengthen the notion that lead disrupts a selected set of cognitive functions.

Although it is commonly recognised that the neurobehavioural effects of lead may be progressive, deficits are usually explored using cross sectional designs. A longitudinal or prospective design, in which a worker is repeatedly examined over a period of time, is more useful for charting functional decline because it is possible to identify early indicators of disturbance, the time course over which disturbed functioning responds to changes in exposure, and whether long term and recent exposure exert similar or different effects on cognition. It is unfortunate, 
therefore, that these designs have rarely been used to study the cognitive changes associated with lead. For occupational studies, the method of choice is to examine workers before exposure to lead occurs and to monitor their progress at regular intervals. In one study, new recruits to lead work were assessed before exposure began and after two years of lead work. ${ }^{9}$ During that period, blood lead concentrations rose and a deterioration on tests of short term memory (digit span) and sensory motor functions (Sana Ana and block design) were noted. In another study, workers were tested over a three year period during which improved conditions of factory hygiene reduced average blood lead concentrations by about $13 \mu \mathrm{g} / \mathrm{dl}$ for the most exposed workers. ${ }^{10}$ Apart from the suggestion of improved self reports of mood, however, no changes were found in psychological test performance. In our earlier cross sectional study, ${ }^{6}$ lead was reported to exert a strong influence on sensory motor functions but only a weak influence on processing and memory functions. The purpose of this paper is to extend our initial analysis of these lead workers by reporting the results from an eight month prospective study during which time blood lead concentrations remained fairly stable.

\section{Design and protocol}

Subjects attended three test sessions at intervals of about four months. On each session they completed a mood adjective checklist, ${ }^{11}$ a work demands and discretion checklist, ${ }^{12}$ and a series of cognitive tasks. ${ }^{6}$ Concurrently with the cognitive testing the concentrations of blood lead and zinc protoporphyrin (ZPP) and urinary aminolaevulinic acid (ALA) were measured as described earlier. ${ }^{6}$ Testing took place on site in a quiet room and subjects received their normal pay during participation.

The test procedure in each session was identical to that described elsewhere. ${ }^{6}$ Subjects performed the following series of microcomputer controlled cognitive tasks: syntactic reasoning (six minutes), delayed five choice reaction time (nine minutes), category search (two minutes), visual spatial memory (five minutes), and category search recall (two minutes). For the category search task different categories were used in each session-namely, birds, fish, and insects. The related distractors were noncategory animal nouns, a third of which were replaced on each session by other animal nouns. The same list of high frequency unrelated distractor nouns was used at each session. As accuracy for all tasks was generally high, the arcsine transformation was applied before analysis.

\section{SUBJECTS}

Seventy male workers completed the study. Their mean age was 41 and they had varying degrees of exposure to lead. Although the study initially aimed to follow up the lead workers for a year (four sessions), due to unavoidable scheduling difficulties complete data could only be collected for the first three sessions. Of the 91 subjects who attended the first session, 74 completed the second session and 70 completed the third session. The main reason for non-attendence was redundancy (10 workers), although five workers had only agreed to participate in the first session, four were not at work during one of the study periods, and two refused to be retested. Each of the remaining 70 subjects was allocated to one of three lead exposed groups on the basis of their average blood lead concentration during the eight month study-namely, low (below $20 \mu \mathrm{g} / \mathrm{dl}$ ), medium (21-40 $\mu \mathrm{g} / \mathrm{dl})$, or high (41-80 $\mu \mathrm{g} / \mathrm{dl})$.

\section{Results}

Table 1 shows the mean values for the three lead groups on several exposure and non-exposure variables. In common with our earlier results ${ }^{6}$ the low lead group had drunk less alcohol in the preceding 24 hours $(F(2,67)=5.3, p<0.05)$ and tended to rate their work as less demanding $(\mathrm{F}(2,67)=3 \cdot 0, \mathrm{p}=$ 0.06). The three lead groups, however, no longer differed significantly in age $(\mathrm{F}(2,67)=1 \cdot 2, \mathrm{p}=0.30)$ indicating that the younger workers tended to be lost from the high lead group. All non-exposure variables shown in table 1 were initially considered eligible for entry as covariates in a repeated measures analysis of variance (ANOVA) with covariance. ${ }^{13}$ The ANOVA consisted of one between subject factor (low, medium, or high lead group) and the main within subject factor was the time of the three test sessions (zero, four, and eight months). All other ANOVA

Table 1 Summary of the mean values on several exposure and non-exposure variables for 70 subjects differentially exposed to lead: averaged over the study

\begin{tabular}{|c|c|c|c|}
\hline & \multicolumn{3}{|c|}{ Lead group } \\
\hline & $\begin{array}{l}\text { Low } \\
(n=\end{array}$ & $\begin{array}{l}\text { Medium } \\
(n=22)\end{array}$ & $\begin{array}{l}\text { High } \\
(n=22)\end{array}$ \\
\hline $\begin{array}{l}\text { Exposure variables: } \\
\text { Blood lead }(\mu \mathrm{g} / \mathrm{dl}) \\
\text { ZPP (mg/dl) } \\
\text { Urinary ALA (mg/l) } \\
\text { Years of lead exposure }\end{array}$ & $\begin{array}{r}14 \cdot 1 \\
13 \cdot 2 \\
2 \cdot 0 \\
7 \cdot 4\end{array}$ & $\begin{array}{r}30.8 \\
33.2 \\
3.3 \\
9.7\end{array}$ & $\begin{array}{r}51.8 \\
77.4 \\
5.9 \\
10.9\end{array}$ \\
\hline $\begin{array}{l}\text { Non-exposure variables: } \\
\text { Age }(\mathrm{y}) \\
\text { Age left school }(\mathrm{y}) \\
\text { Normal sleep }(\mathrm{h}) \\
\text { Recent sleep }(\mathrm{h})^{\star} \\
\text { Weekly alcohol intake }(\mathrm{ml}) \dagger \\
\text { Recent alcohol intake }(\mathrm{ml})^{\star} \\
\text { Work demands } \\
\text { Work discretion } \\
\text { Stress score } \\
\text { Arousal score }\end{array}$ & $\begin{array}{r}43 \cdot 9 \\
15 \cdot 5 \\
7 \cdot 4 \\
7 \cdot 1 \\
200 \cdot 0 \\
6 \cdot 0 \\
14 \cdot 2 \\
16 \cdot 4 \\
3 \cdot 9 \\
9 \cdot 1\end{array}$ & $\begin{array}{r}41 \cdot 0 \\
15 \cdot 4 \\
7 \cdot 1 \\
7 \cdot 0 \\
290 \cdot 0 \\
26 \cdot 0 \\
15 \cdot 9 \\
15 \cdot 4 \\
3 \cdot 9 \\
7 \cdot 8\end{array}$ & $\begin{array}{r}39 \cdot 1 \\
15 \cdot 2 \\
6 \cdot 8 \\
6 \cdot 6 \\
250 \cdot 0 \\
33 \cdot 0 \\
17 \cdot 1 \\
14 \cdot 3 \\
3 \cdot 5 \\
7 \cdot 9\end{array}$ \\
\hline
\end{tabular}

$\star$ Value for the past 24 hours.

$\dagger 20 \mathrm{ml}=1$ pint beer. 
Table 2 Mean concentrations of blood lead ( $P b B$ in $\mu g / d l)$ and $Z P P(\mu \mathrm{g} / \mathrm{dl})$ and urinary $A L A(A L A$ in $\mathrm{mg} / \mathrm{l})$ at four monthly intervals during the eight month study

\begin{tabular}{|c|c|c|c|c|c|c|c|c|c|}
\hline \multirow[b]{3}{*}{ Session } & \multicolumn{9}{|c|}{ Lead group } \\
\hline & \multicolumn{3}{|l|}{ Low } & \multicolumn{3}{|c|}{ Medium } & \multicolumn{3}{|l|}{ High } \\
\hline & $P b B$ & $Z P P$ & $A L A$ & $P b B$ & $Z P P$ & $A L A$ & $P b B$ & $Z P P$ & $A L A$ \\
\hline $\begin{array}{l}\text { One } \\
\text { Two } \\
\text { Three }\end{array}$ & $\begin{array}{l}15 \cdot 0 \\
13 \cdot 2 \\
14 \cdot 2\end{array}$ & $\begin{array}{l}12.7 \\
14.9 \\
11.8\end{array}$ & $\begin{array}{l}2.8 \\
1.6 \\
1.7\end{array}$ & $\begin{array}{l}32.3 \\
30 \cdot 2 \\
30 \cdot 0\end{array}$ & $\begin{array}{l}30 \cdot 1 \\
43.5 \\
26 \cdot 1\end{array}$ & $\begin{array}{l}3 \cdot 7 \\
3 \cdot 3 \\
3 \cdot 0\end{array}$ & $\begin{array}{l}50.8 \\
54.0 \\
50.6\end{array}$ & $\begin{array}{l}75 \cdot 9 \\
83.5 \\
72 \cdot 8\end{array}$ & $\begin{array}{l}6.4 \\
5.8 \\
5.6\end{array}$ \\
\hline Mean & $14 \cdot 1$ & $13 \cdot 1$ & 2.0 & $30 \cdot 8$ & $33 \cdot 2$ & $3 \cdot 3$ & $51 \cdot 8$ & $77 \cdot 4$ & 5.9 \\
\hline
\end{tabular}

variables were within subject factors specific to the structure of each cognitive task. When significant correlations between covariates and performance were found, the influence of the relevant covariates was controlled before considering that any differences were due to lead. These results are reported under the effects of factors other than exposure. The correlations between absorption of lead and performance were computed using multiple regression techniques and, unless specified, the measures used are the average values during the study.

Table 2 shows that group levels of lead absorption remained at a fairly constant value during the eight month study. A series of two way ANOVAs, however, gave the following results: (1) a group $\mathrm{x}$ session interaction $(F(4,134)=4 \cdot 1, p=0.004)$ for blood lead concentration, (2) slightly raised concentrations of ZPP at the second session $(F(2,134)=2 \cdot 6$, $\mathrm{p}=0.08)$ that did not vary as a function of lead group $(\mathrm{p}=0.79)$, and (3) a small decline in urinary ALA concentrations during the study $(F(2,134)=3 \cdot 4$, $p$ $=0.04)$. The main effect of lead group was of course highly significant for each measure of lead absorption. As table 2 shows, the blood lead interaction reflects the greater separation between the low and high lead groups at the second test session.

Table 3 shows the absolute value (modulus) of the session to session changes, averaged for each lead group, in the three measures of lead absorption. The average changes for blood lead and urinary ALA concentrations were small for all groups, but ZPP concentrations for the medium and high lead groups

Table 3 Average absolute values of session to session changes (modulus) in individual levels of lead absorption during the eight month study (units as in table 2)

\begin{tabular}{|c|c|c|c|c|c|c|c|}
\hline \multirow[b]{3}{*}{ Session } & \multicolumn{7}{|c|}{ Lead group } \\
\hline & \multicolumn{3}{|l|}{ Low } & \multicolumn{2}{|c|}{ Medium } & \multicolumn{2}{|l|}{ High } \\
\hline & $P b B$ & $Z P P$ & D $A L A$ & $P b B$ & $Z P P A L A$ & $P b B$ & $Z P P A L A$ \\
\hline $\begin{array}{l}\text { One-two } \\
\text { Two-three } \\
\text { One-three }\end{array}$ & $\begin{array}{l}2 \cdot 5 \\
2 \cdot 0 \\
2 \cdot 3\end{array}$ & $\begin{array}{l}6 \cdot 2 \\
4 \cdot 1 \\
3 \cdot 9\end{array}$ & $\begin{array}{l}2 \cdot 1 \\
0.5 \\
1.9\end{array}$ & $\begin{array}{l}4.9 \\
3.5 \\
4.7\end{array}$ & $\begin{array}{rr}12.7 & 1.9 \\
7.6 & 1.4 \\
8.0 & 1.5\end{array}$ & $\begin{array}{l}6 \cdot 1 \\
5 \cdot 7 \\
4 \cdot 4\end{array}$ & $\begin{array}{ll}20.0 & 2.3 \\
20.3 & 2.8 \\
17.6 & 3.6\end{array}$ \\
\hline
\end{tabular}

showed wider fluctuations. To determine whether the cognitive performance of a subject changed concurrently with a change in lead absorption, these correlations were assessed using within subject covariance analysis. ${ }^{13}$

\section{MOOD CHECKLIST}

After significant covariates had been controlled, the three groups did not differ in self reported stress $(p=0 \cdot 13)$ and stress scores were not correlated with either average blood lead concentrations $(p=0 \cdot 14)$ or blood lead concentration changes during the study $(p=0.85)$. Similarly, concentrations of blood ZPP and urinary ALA were not correlated with stress levels.

By contrast, after the influence of stress and work discretion had been controlled, self reported arousal correlated negatively with blood lead concentrations $(\mathrm{r}=-0.23, \mathrm{~T}=-2.3, \mathrm{p}=0.02)$; but the correlation was eliminated once work demands were forced into the regression equation (see below). Variations in blood lead, ZPP and urinary ALA concentrations during the study were not correlated with changes in arousal.

\section{Effects of other factors}

Workers reported higher arousal when self reported stress was lower $(\mathrm{r}=-0.45, \mathrm{~T}=-4.4, \mathrm{p}=0.001)$ and vice versa $(\mathrm{r}=-0.51, \mathrm{~T}=-4.9, \mathrm{p}<0.001)$. Arousal was higher when subjects rated their work as high in autonomy or discretion $(r=0.32, T=3.1$, $p=0.003)$ and low in demands $(r=-0.21$, $\mathrm{T}=-1.95, \mathrm{p}=0.06$ ).

After significant covariates had been controlled, entering blood lead concentration into the regression equation $(r=-0.18, p=0.12)$ eliminated the correlation between arousal and work demands $(r=-0.13, p=0.29)$ and the subsequent stepwise removal of work demands showed lower arousal with increasing blood lead concentration $(r=-0.23$, $p=0.02)$ as reported above. The interrelation between arousal, work demands, and blood lead concentrations was equivalent to that reported before $^{6}$ and although work demands influence arousal in other occupation $\mathrm{s}^{14}$ it was possible that controlling for arousal in this situation might attenuate putative performance effects of lead. In common with our earlier analysis strategy it was decided not to control initially for the influence of arousal on performance, but self reported work demands would be controlled, when necessary, by covariate analysis.

\section{SYNTACTIC REASONING}

Table 4 summarises the overall improvements in syntactic reasoning time and accuracy during the study. For performance changes during the six minute task, the only effect of exposure to emerge was the group $\mathrm{x}$ work duration interaction $(\mathrm{F}(4,134)=$ 
Table 4 Improvements in overall syntactic reasoning time (s) and accuracy (\%) for the three lead groups during the study (accuracy in parentheses)

\begin{tabular}{llll}
\hline & \multicolumn{2}{l}{ Lead group } & \\
\cline { 2 - 4 } Session & Low & Medium & High \\
\hline One & $5 \cdot 08(88)$ & $4 \cdot 86(84)$ & $5.41(85)$ \\
Two & $4.82(92)$ & $4.97(84)$ & $4.98(83)$ \\
Three & $4.38(92)$ & $4.59(90)$ & $4 \cdot 77(84)$ \\
\hline
\end{tabular}

$2 \cdot 7, \mathrm{p}=0.03):$ the low and medium lead groups showed improved reasoning times during the task, but the high lead group did not. Reasoning accuracy remained constant during the task for all groups.

Turning to the linguistic factors, six subjects in the high lead group, three in the medium lead group, and one in the low lead group had $0 \%$ accuracy on certain problems (mainly passives) at some point during the study. Due to the missing correct reaction time data for that linguistic condition their results had to be excluded from analysis. In common with other work $^{61415}$ highly reliable influences of statement voice and negation on both speed and accuracy, and a truth $x$ negation interaction were found (all $p<0.001$ ) that is, the reasoning problems varied systematically and substantially in terms of their difficulty. Reasoning times improved with practice $(\mathrm{F}(2,114)=15 \cdot 0$, $\mathrm{p}<0.001)$ and this was especially so for the difficult true negative problems $(F(2,114)=4 \cdot 0, p=0 \cdot 02)$. In general, only the accuracy of solving passive problems improved with practice $(\mathrm{F}(2,114)=4 \cdot 4$, $\mathrm{p}=0.02$ ).

For exposure to lead, the critical truth $\mathrm{x}$ voice $\mathrm{x}$ negation $x$ group interaction reported in our earlier analysis ${ }^{6}$ tended to vary during the study $(F(4,114)$ $=2 \cdot 2, \mathrm{p}=0.07)$ such that the initial impairment of the high lead group on the complex problems was eliminated by practice. A series of ANOVAs confirmed the presence of the four way interaction on the first session, at about the same level of significance previously seen $(F(2,57)=2 \cdot 5, p=0.09)$, but not on the second $(p=0.65)$ and third $(p=0.70)$ session. It can be concluded that the initial lead impairment disappears with practice. Figure 1 shows that on the final session the high lead group was not differentially slowed by problem complexity and the difference between the low and high lead groups $(0.48 \mathrm{~s})$ was not significant. It is worth stating that the complexity effect did not manifest itself on any other aspect of reasoning speed on the second and third sessions.

For accuracy (table 4), when all subjects were considered $(n=70)$, the only effect of lead was the failure of the high lead group to improve with practice; the group $\mathbf{x}$ session interaction gave $F(4,133)=3.6, p=0.008$. This interaction remained significant when subjects with $0 \%$ accuracy on certain problems were excluded from analysis
$(\mathrm{F}(4,113)=4 \cdot 1, \mathrm{p}=0.004)$. Multiple regression showed that blood lead concentration correlated with overall accuracy $(\mathrm{r}=-0.24, \mathrm{~T}=-2 \cdot 2, \mathrm{p}=0.03)$, but this was due to the contribution from the second $(\mathrm{r}=-0.30, \mathrm{p}=0.008)$ and third $(\mathrm{r}=-0.26$, $\mathrm{p}=0.02$ ) sessions; the correlation was insignificant on the first session $(r=-0 \cdot 13, p=0 \cdot 27)$. None of the correlations with ZPP or urinary ALA concentration approached significance. This pattern of correlations is consistent with the group $\mathbf{x}$ session interaction: practice did not improve the accuracy of the high lead group and larger group differences were seen following practice. These results imply that, unlike the low and medium lead groups, the improved reasoning time of the high lead group was to some extent achieved at the cost of a failure to improve accuracythat is, a speed accuracy tradeoff in the direction of less caution.

\section{Effects of other factors}

Reasoning accuracy was poorer when stress was high $(\mathrm{r}=-0.39, \mathrm{~T}=-3.49, \mathrm{p}<0.001)$ and correct reasoning time was faster when arousal was high $(r=0 \cdot 27, T=-2 \cdot 27, p=0.03)$. Controlling for the influence of arousal did not alter the results reported above.

CATEGORY SEARCH AND FREE RECALL

Original classification of nouns at time of presentation Table 5 shows the influence of the semantic variables on classification time and accuracy. For nouns not in the category (distractors), unrelated distractors were

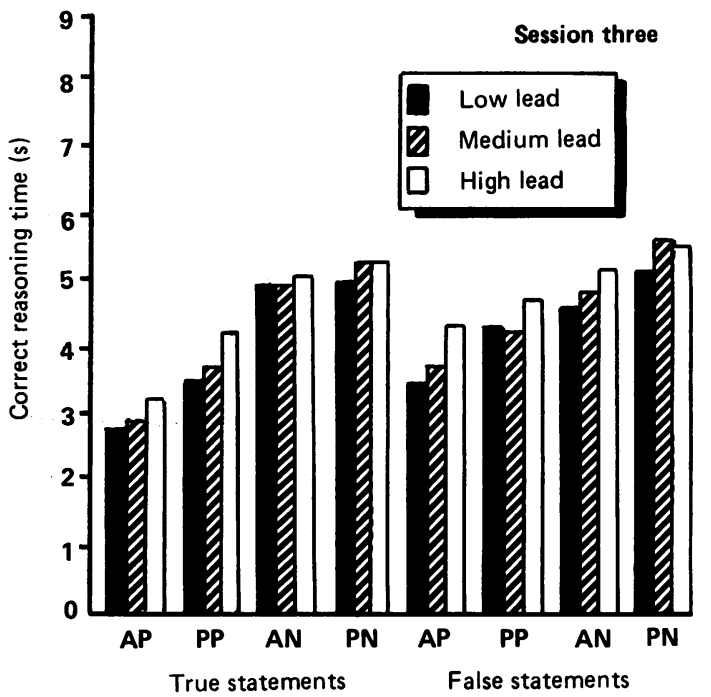

Figure 1 Correct syntactic reasoning times (s) on the third session as a function of problem complexity. Active positive $(A P)$, passive positive $(P P)$, active negative $(A N)$, and passive negative ( $P N)$. 
Table 5 Noun classification time (ms) and accuracy (\%) as a function of lead exposure: averaged across sessions (accuracy in parentheses)

\begin{tabular}{llll}
\hline & \multicolumn{2}{l}{ Lead group } & \\
\cline { 2 - 4 } & Low & Medium & High \\
\hline Typical instance & $1017(99 \cdot 9)$ & $1015(99 \cdot 9)$ & $1182(99 \cdot 9)$ \\
Atypical instance & $1227(87 \cdot 0)$ & $1209(87.0)$ & $1410(87 \cdot 5)$ \\
Related distractor & $1280(99 \cdot 4)$ & $1195(99 \cdot 2)$ & $1386(97 \cdot 5)$ \\
Unrelated distractor & $1137(99 \cdot 9)$ & $1093(99 \cdot 9)$ & $1349(99 \cdot 6)$ \\
\hline
\end{tabular}

rejected faster and more accurately $(p<0.001)$ than related distractors. For the category nouns: (1) typical instances were classified faster and more accurately $(p<0.001)$ than atypical instances, (2) category decisions were slowed down by the presence of related, compared to unrelated, distractors $(\mathbf{p}=$ 0.002 ), and (3) this slowing was greater for atypical category instances $(p=0.02)$. These "semantic complexity" findings are identical to those reported before. ${ }^{6}$ Finally, the effect of typicality was stronger for both speed and accuracy on the second and third sessions, when the search categories were fish and insects (typicality $\times$ session interaction; both $\mathrm{p}<0.001)$.

The high lead group took longer to reject distractor nouns $(F(2,66)=2 \cdot 5, p=0.09)$ and accept category nouns $(p=0 \cdot 12)$, but neither result achieved acceptable significance and none of the correlations with blood lead, ZPP, and urinary ALA concentrations was significant. For the category nouns, no interactions were found between the semantic factors and lead group. For the distractors, the group $\times$ distractor interaction $(F(2,66)=3.3, p=0.04)$ showed that the high lead group took disproportionately longer to reject unrelated distractors (table 5). Although unrelated distractors were easier to reject than related distractors, the correlation between blood lead concentrations and rejection time was of borderline significance for unrelated distractors $(r=$ $0 \cdot 20, T=1 \cdot 7, p=0.09)$ and insignificant for related distractors $(r=0.09, p>0.25)$.

Although lead did not impair the accuracy of accepting category nouns, the high lead group rejec-

Table 6 The mean number of nouns correctly and incorrectly recalled in the delayed free recall task: averaged over all sessions

\begin{tabular}{llll}
\hline & \multicolumn{2}{l}{ Lead group } & \\
\cline { 2 - 4 } & Low & Medium & High \\
\hline Typical instance & 4.39 & 4.33 & 4.35 \\
Atypical instance & 3.42 & 3.13 & 2.86 \\
Related distractor & 2.12 & 2.58 & 2.21 \\
Unrelated distractor & 1.77 & 1.13 & 0.96 \\
Intrusion error & 0.25 & 0.12 & 0.21 \\
Extra list error & 0.68 & 0.89 & 0.79 \\
\hline
\end{tabular}

ted distractors less accurately $(\mathrm{F}(2,66)=3.34, \mathrm{p}=$ 0.04 ), the correlation with blood lead concentration was significant $(r=-0.30, T=-2.53, p=0.01)$, and the correlation was higher for the unrelated $(\mathbf{r}=-0.35, \mathrm{p}=0.003)$ than the related $(r=-0.27, p=0.03)$ distractors. Other than a borderline correlation between urinary ALA concentration and accuracy for unrelated nouns $(r=-0 \cdot 22$, $p=0.07)$ none of the ZPP or ALA correlations was significant. These results imply that the high lead group are achieving their speed at the expense of poorer accuracy. This speed-accuracy tradeoff is similar to that reported before, ${ }^{6}$ except that over time the more exposed workers move in the direction of sacrificing accuracy for speed-that is, they become less cautious.

\section{Results on the delayed free recall of nouns}

Subjects correctly recalled about 11 nouns from the classification task (table 6) and, as expected, recall levels declined progressively in the order: typical instances, atypical instances, related distractors, and unrelated distractors $(F(3,198)=145 \cdot 0, p<0.001)$. That is, the nouns varied substantially in terms of their ease of recall. Across the three sessions the recall of typical instances remained constant $(p=0.27)$ whereas the recall of atypical instances declined $(F$ $(2,132)=6 \cdot 1, p=0.003)$ in line with their poorer accuracy during initial classification.

As noted, certain distractor nouns were repeated across sessions: all unrelated distractors were repeated whereas only two thirds of the related distractors were repeated. Although four months separated the test sessions, as expected, the recall of unrelated distractors improved with repeated presentation $(F(2,132)=10.5, p<0.001)$. This improvement can be considered indicative of long term incidental learning or the adoption of better recall strategies. The recall of related distractors showed a similar improvement across sessions $(\mathrm{p}<$ 0.01 ) and, as expected from the related distractor manipulations, this was accompanied by the increased production of nouns presented only on an earlier session-that is, intrusion errors increased over sessions $(F(2,132)=11.3, p<0.001)$. The production of nouns never presented (extra list errors) did not vary during the study.

Exposure to lead did not impair the recall of category nouns, either typical or atypical instances, but it did impair the recall of distractor nouns. For these nouns, a group $\times$ distractor interaction (F $(2,66)=5.9, \mathrm{p}=0.004)$ showed that the high lead group recalled fewer unrelated distractors and an equivalent number of related distractors. Multiple regression confirmed this: blood lead concentration correlated with the recall of unrelated distractors ( $r$ $=-0.33, \mathrm{~T}=-2.9, \mathrm{p}=0.006)$ but not related distractors $(r=-0.04, p=0.71)$. Of some interest 


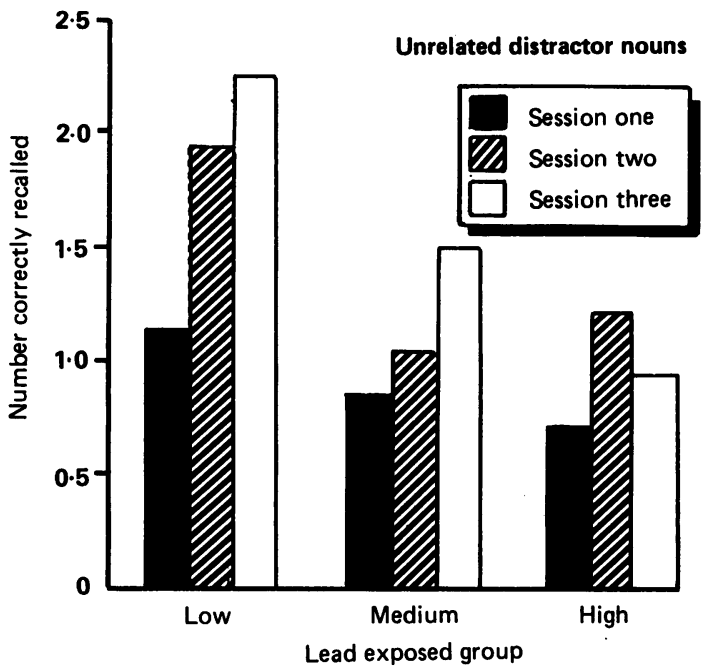

Figure 2 Improvements over sessions in the recall of unrelated distractors as a function of lead exposure.

was that concentrations of blood ZPP $(r=-0 \cdot 23, T$ $=-1.9, \mathrm{p}=0.056)$ and urinary $\operatorname{ALA}(\mathrm{r}=-0.32, \mathrm{~T}$ $=-2 \cdot 8, p=0.007)$ also correlated only with the recall of unrelated distractor nouns. It can be concluded that exposure to lead does not impair the recall of nouns that formed the focus of the search (category nouns), nor does it impair the recall of nouns related to that focus (related distractors), but lead does impair, in an exposure-response manner, the recall of nouns poorly related to the search category (unrelated distractors). This impairment is indexed by all three measures of lead absorption.

This impaired ability of the high lead group to recall unrelated distractors further interacted with the test session $(F(4,132)=2 \cdot 6, p=0.04)$ implying a non-uniform lead impairment during the study. Figure 2 shows that the low lead group improved their recall of unrelated distractors throughout the study. By contrast, those with higher lead exposure, particularly the high lead group, did not benefit to the same extent. The lead impairment seen on the first session is therefore magnified on subsequent sessions.

\section{Effects of other factors}

None of the covariates correlated significantly with classification time or accuracy but age was negatively correlated with the recall measures. Multiple regression showed that age did not influence the recall of typical nouns $(r=-0.18, p=0.14)$, but older subjects recalled fewer atypical nouns $(r=-0 \cdot 23, p$ $=0.05)$, related distractors $(\mathrm{r}-0.31, \mathrm{p}=0.009)$, and unrelated distractors $(\mathrm{r}=-0.24, \mathrm{p}=0.04)$.
DELAYED SERIAL FIVE CHOICE REACTION TIME

Considering first the influence of task parameters, decision times were slower $(F(3,201)=14 \cdot 0, p<$ 0.001 ) and the decision gap rate was higher $(F(3,201)=31.8, p<0.001)$ at short durations of waiting for light presentation. By contrast, movement times were faster $(F(3,201)=18 \cdot 3, p<$ 0.001 ) and the movement gap rate was lower $(\mathrm{F}(3,201)=6.1, \mathrm{p}<0.001)$ at short durations. Figure 3 shows that blood lead concentration was correlated with slower decision times $(r=0.27$, $\mathrm{T}=2.4, \mathrm{p}=0.02)$ and movement times $(\mathrm{r}=0.32$, $T=2 \cdot 86, p=0.006)$ and this slowing was independent of the duration of waiting. Multiple regression confirmed that blood lead concentration was correlated with (a) decision times at each duration of waiting (0-1s: $r=0.31, p=0.01 ; 1-2 s: r=0.28, p=$ $0.02 ; 2-3 \mathrm{~s}: \mathrm{r}=0.24, \mathrm{p}=0.03 ; 3-4 \mathrm{~s}: \mathrm{r}=0.24, \mathrm{p}=$ $0.04)$ and (b) movement times at each duration of waiting (0-1s: $r=0.34, p=0.003 ; 1-2 s: r=0.33, p$ $=0.005 ; 2-3 \mathrm{~s}: \mathrm{r}=0.32, \mathrm{p}=0.006 ; 3-4 \mathrm{~s}: \mathrm{r}=0.29, \mathrm{p}$ $=0.01$ ). These findings confirm those reported previously ${ }^{6}$ and table 7 shows the mean performance levels for the three groups averaged across the duration of waiting factor.

Although the three groups did not differ in the incidence of movement gaps $(F<1)$, the group $x$ duration of waiting interaction for decision gaps ( $F$ $(6,201)=3.3, p=0.004)$ showed increasing impairment for the medium and high lead groups at shorter durations (fig 4). Multiple regression analyses showed a significant correlation between decision gap rates and blood lead concentration at the shortest period of waiting (0-1s: $r=0.32, p=0.007)$, but not thereafter (1-2s: $r=0 \cdot 18, p=0 \cdot 14 ; 2-3 s: r=0 \cdot 12, p$ $0.33 ; 3-4 s: r=-0.09, p=0.49)$. None of the correlations with ZPP and ALA concentration was significant.

Peformance on the task did not vary substantially in magnitude during the eight month study. Decisions times slowed by $40 \mathrm{~ms}(\mathrm{~F}(2,133)=9 \cdot 1, \mathrm{p}$ $<0.001)$ and movement times were $15 \mathrm{~ms}$ slower on the second session $(F(2,134)=3 \cdot 1, p=0.05)$; but none of the interactions with lead exposure was

Table 7 Summary of various performance measures in the five choice reaction time task as a function of lead exposure: averaged across sessions

\begin{tabular}{|c|c|c|c|}
\hline & \multicolumn{3}{|c|}{ Lead group } \\
\hline & Low & Medium & High \\
\hline $\begin{array}{l}\text { Error rate }(\%) \\
\text { Decision time (ms) } \\
\text { Decision variability (ms) } \\
\text { Decision gaps }(\%) \\
\text { Movement time (ms) } \\
\text { Movement variability (ms) } \\
\text { Movement gaps }(\%)\end{array}$ & $\begin{array}{c}1 \cdot 2 \\
748 \\
177 \\
9 \cdot 5 \\
312 \\
48 \\
1 \cdot 2\end{array}$ & $\begin{array}{c}1 \cdot 7 \\
748 \\
165 \\
10 \cdot 5 \\
304 \\
39 \\
1 \cdot 2\end{array}$ & $\begin{array}{c}1 \cdot 2 \\
826 \\
187 \\
12 \cdot 8 \\
362 \\
47 \\
1 \cdot 1\end{array}$ \\
\hline
\end{tabular}




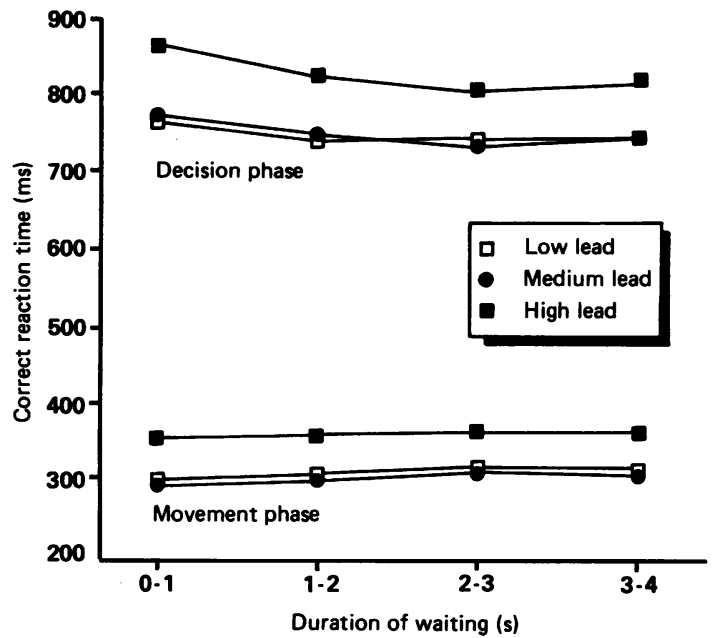

Figure 3 Movement and decision times (ms) as a function of duration of waiting for light presentation (s) and lead exposure.

significant. Of some interest, the analysis of covariance showed a borderline relation between fluctuations in individual ZPP concentrations and concurrent changes in decision time $(F(1,132)=3 \cdot 7$, $\mathrm{p}=0.06$ ) such that a rise in ZPP concentration was associated with a slower decision time and vice versa. The equivalent correlations for blood lead $(p=0.24)$ and urinary ALA concentrations $(p=0 \cdot 11)$ were not significant.

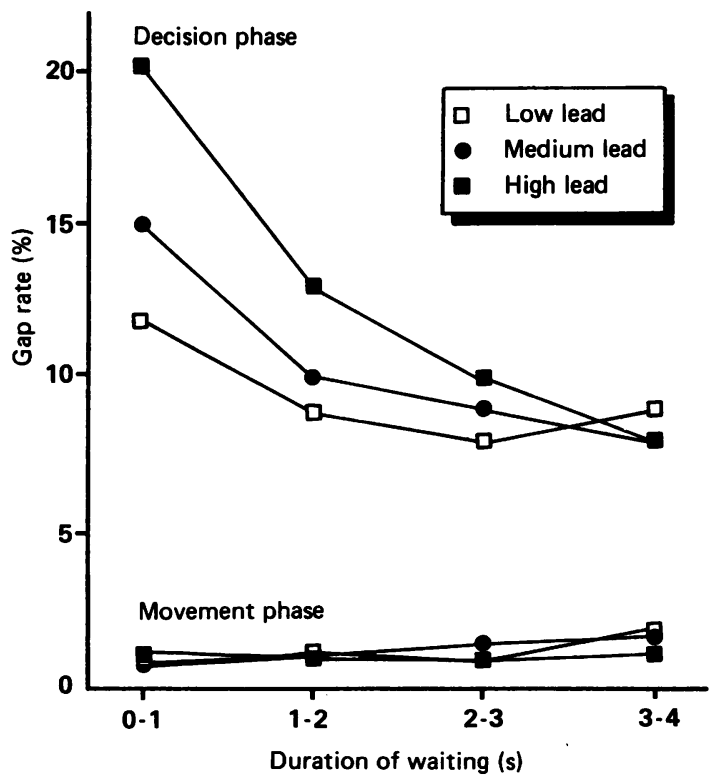

Figure 4 Movement and decision gap rates (\%) as a function of duration of waiting for light presentation (s) and lead exposure.
Effects of other factors

Covariate analysis showed that age was correlated with slower $(\mathrm{r}=0.34, \mathrm{~T}=3.1, \mathrm{p}=0.003)$ and more variable $(\mathrm{r}=0.28, \mathrm{~T}=2 \cdot 4, \mathrm{p}=0.02)$ decision times, slower $(r=0.36, T=3.2, p=0.002)$ and more variable $(\mathrm{r}=0.22, \mathrm{~T}=1.9, \mathrm{p}=0.06)$ movement times, higher decision gaps rates $(r=0 \cdot 21, T=1 \cdot 8$, $p=0.07)$, and higher error rates $(r=0.29, T=2.6$, $p=0.01)$. Finally, workers reporting higher levels of work autonomy took longer to make decisions $(\mathbf{r}=$ $0 \cdot 24, \mathrm{~T}=2 \cdot 2, \mathrm{p}=0.03$ ).

\section{VISUAL SPATIAL RECOGNITION MEMORY}

Data from two subjects in the low lead group and one subject in the high lead group had to be excluded from analysis mainly because of $0 \%$ accuracy with memory set sizes of six. On average the remaining 67 subjects recognised the position of the probe with $86 \%$ accuracy with a correct reaction time of $1.32 \mathrm{~s}$. Probe recognition was slower $(F(2,128)=56 \cdot 2, p<$ $0.001)$ and less accurate $(F(2,128)=163, p<0.001)$ as the number of locations to remember increased; with a faster decline in the accuracy of identifying false probes compared with true probes $(F(2,128)=$ $8.4, p<0.001)$. Recognition times tended to improve across sessions $(F(2,127)=2.5, p=0.09)$, but with no improvement in accuracy $(F<1)$. The three lead groups did not differ in the speed of spatial recognition (table 8) and, after controlling covariates (see below), none of the exposure-response relations was significant. Blood lead concentration tended to correlate with poorer recognition accuracy ( $r$ $=-0.20, \mathrm{~T}=-1.74, \mathrm{p}=0.09$ ) when work autonomy was controlled and the blood lead correlation improved when work autonomy was removed ( $r$ $=-0 \cdot 25, \mathrm{~T}=-2 \cdot 1, \mathrm{p}=0.04)$. None of the interactions between exposure to lead and the cognitive variables (for example, memory set size), however, approached significance. A reanalysis of the accuracy data, which included data from subjects with missing recognition times, did not uncover any effects of exposure to lead.

\section{Effects of other factors}

Covariate analysis showed that age was correlated with longer recognition times $(r=0 \cdot 36, T=3 \cdot 1, p$ $=0.003)$. Workers reporting higher work demands

Table 8 Changes in overall visual spatial recognition time (ms) and accuracy (\%) as a function of lead exposure and test session (accuracy in parentheses)

\begin{tabular}{llll}
\hline & \multicolumn{3}{l}{ Lead group } \\
\cline { 2 - 4 } Session & Low & Medium & High \\
\hline One & $1340(85)$ & $1287(85)$ & $1442(84)$ \\
Two & $1264(87)$ & $1294(89)$ & $1362(84)$ \\
Three & $1245(88)$ & $1291(86)$ & $1364(83)$ \\
\hline
\end{tabular}


were slower $(r=0.25, T=2.2, p=0.04)$ whereas those reporting higher work autonomy were more accurate $(\mathrm{r}=0.32, \mathrm{~T}=2 \cdot 8, \mathrm{p}=0.007)$. Excluding the work demand covariate from the recognition time analysis gave a borderline correlation with blood lead concentration $(\mathrm{r}=0.21, \mathrm{~T}=1.8, \mathrm{p}=0.08)$ but did not otherwise modify the results.

\section{Discussion}

The present study examined the cognitive functioning of lead workers over an eight month exposure period when biochemical indicators of lead absorption remained stable. In general terms, the findings support the view that workers whose blood concentrations are usually higher than $40 \mu \mathrm{g} / \mathrm{dl}$, the high lead group, are impaired on many psychological tasks. ${ }^{1-6}$ Those workers with blood lead concentrations of $21-40 \mu \mathrm{g} / \mathrm{dl}$, the medium lead group, showed little evidence of impairment. The task impairments found usually correlated best with a worker's average blood lead concentration over the preceding eight months and only rarely with concentration of ZPP and urinary ALA. In line with biochemical measures of lead absorption, the impairments in performance tended to remain stable during the study and this implies that neither practice at the tasks, nor continued exposure to lead, altered the findings. The high lead group, however, also show changes over the study consistent with more "risky" responding.

In common with our earlier cross sectional analysis, this study found that the functional locus for much of the task disruption centred around the sensory motor requirements of the task. The clearest evidence was seen in the five choice task and, after excluding extra slow reaction times (gaps), blood lead concentration correlated with long movement times and long decision times. For the high lead group, decision time $(78 \mathrm{~ms})$ was slowed more than movement time $(54 \mathrm{~ms})$ and this suggests that whereas motor disruption dominates, processes specific to the decision phase were also affected. In choice reaction time tasks, decision time depends both on the degree of preparation and on the speed of information processing once the signal is detected. The present results show that lead has an equivalent effect at short and at long preparation intervals. This pattern resembles that found for age and alcohol, but not practice, in which reduced information processing rates are implicated in the slowing..$^{16-17}$

Transitory failures in rapidly detecting and responding to lights (decision gaps) were also greater in the high lead group at short preparation times (see fig 4). Previous work ${ }^{1617}$ has shown that the optimal preparation time is about $500 \mathrm{~ms}$ and thus further work with short preparation times is needed to determine explicitly if preparation rate is influenced by exposure to lead and to explore the part played by the control of attention at these short delays. The dominance of the motor component in the slowing of response time accords well with evidence of slowed motor nerve conduction velocities in lead workers, ${ }^{18-21}$ but the relation between the two measures remains unknown. It is also possible that part of the slowing during the decision phase can be traced to sensory encoding processes because lead appears to reduce visual sensitivity by damaging central and peripheral optic nerve fibres. ${ }^{22}$ Such interrelations between the central and peripheral nervous system emphasise that the integrity of cognitive functioning is predicated on the adequate functioning of the supporting neurophysiological processes.

The fact that both sensory motor slowing and increased lapses in concentration were seen throughout the eight month study implies that these dysfunctions form a robust and enduring characteristic of exposure to lead. Moreover, the dysfunctions were not eliminated by practice, which is an important consideration when designing and selecting tasks for prospective studies. More speculatively, the positive correlation between changes in ZPP concentration and decision times could indicate a simple link between absorption of lead over the past few months and current decision time. The failure to find a significant relation of decision time with blood leado concentration may reflect the restricted range of changes which occurred (see table 3 ). Further prospective studies will be required to determine if the influence on decision times represents a dysfunction due to lead that is reversible in the sense of being sensitive to variations in recent absorption of lead.

The second major effect of lead on performance was in a memory task in which subjects recalled nouns classified in an earlier category search task. The general results from the task were consistent with studies showing the importance of initial processing on retention levels. ${ }^{23}{ }^{24}$ The memory deficit associated with exposure was not a global one but was restricted to one type of noun (unrelated distractors). Thus exposure to lead was correlated neither with the recall of category members, nor with the recall of nouns related to the category through an immediate superordinate category, but all three measures of absorption of lead were negatively correlated with the recall of nouns that had a low similarity to the search category. This difficulty with unrelated distractors increased over time. Given that the distractors were repeated across sessions, and that the low lead group showed substantial improvements in recall with such repetition, the result implies that the high lead group either showed poorer long term incidental learning or failed to improve on their recall strategy.

Our present data do not allow a firm choice to be made as to whether this difficulty arises from deficits 
at encoding, retention, or retrieval. The evidence implicating encoding is not strong because the high lead group classified the unrelated distractors with high accuracy and actually spent longer classifying them. This should have ensured comparable encoding levels. With respect to retention, studies manipulating the interval between classification and recall, which was five minutes in the present study, would help resolve whether the impairments are mediated by the more rapid loss of information from memory. Other work, however, has shown that once lead workers have learned material they do not appear to forget it faster. ${ }^{5}$ For retrieval, the inclusion of a test of recognition memory would help to establish if the difficulties arise from poorer recall strategies or memorial organisation since these factors tend to influence measures of recall more than recognition. A further unresolved issue concerns the finding that the major difficulty in both the classification task and recall task is with unrelated distractors. Although it seems reasonable to assume that these two results are connected, no simple relation is apparent.

More generally, the results suggest that lead slows sensory motor reaction time and it is worth recalling that the high lead group had slowed reaction times on all the tasks. It is of some interest, therefore, to examine the extent to which sensory motor slowing contributed to slowing in the other tasks. For example, lead could be associated with a purely sensory motor deficit or it could be accompanied by a central deficit. Figure 5 plots the relation between the correct reaction times of the high and low lead groups at several levels of cognitive complexity from each task using the method suggested by Brinley. ${ }^{25}$

In an unconstrained analysis a two factor linear model described the data set extremely well. (High lead group $\mathrm{RT}(\mathrm{s})=\mathbf{0 . 0 4 8}+\mathbf{1 . 0 7}$ low lead group $\mathrm{RT}$ (s) $\mathrm{r}=0.998, \mathrm{~T}=67.1, \mathrm{p}<0.001$.) The constant and slope of the model can be broadly characterised as the peripheral and central contributions of the lead deficit respectively. ${ }^{26}$ The value of the constant (48 $\mathrm{ms}$ ) is close to the value of the movement time slowing ( $54 \mathrm{~ms}$ ) and the slope is greater than unity implying a mild central slowing factor. The constant failed, however, to differ from zero $(p=0.31)$ and the single factor model, constrained to pass through the origin, showed a simple multiplicative effect on response times. (High lead group RT (s) $=1.08$ low lead group $R T(s) ; r=0.999, T=102.3, p<0.001$.) Although the final equation does not include a peripheral component, it is worth recalling that the major distinction between the choice reaction time task and the other cognitive tasks in motor terms is that the first required a large hand movement $(13 \mathrm{~cm})$ whereas the other tasks only required a small finger movement. It is to be expected, therefore, that the performance impact of a motor slowing will be more easily seen when motor demands dominate.

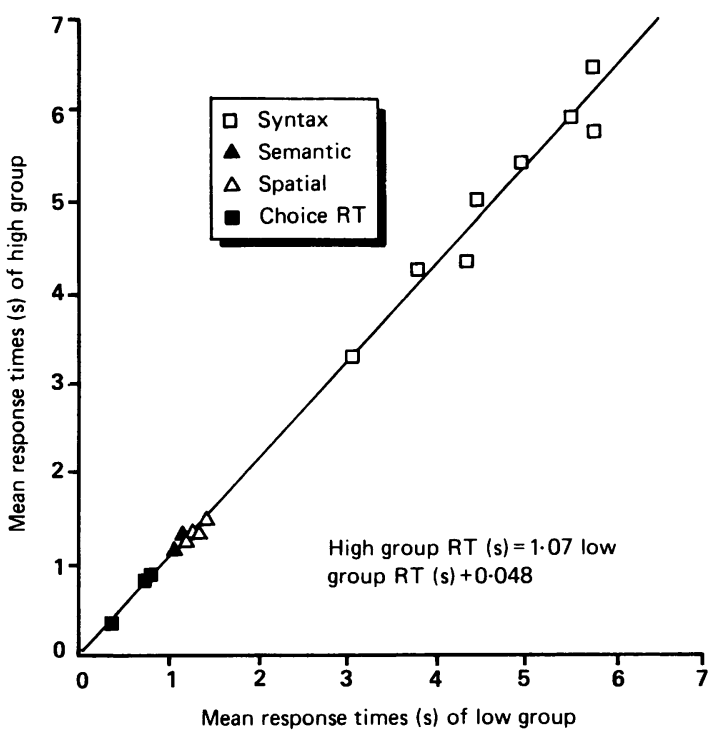

Figure 5 Relation between the average response times $(s)$ of the high lead exposed group and the low lead exposed group across the four cognitive tasks.

The conclusions of the above analysis are based on the average reaction times during the study and this global analysis is likely to be insensitive to subtle alterations in performance. Practice on the verbal reasoning tasks, for example, tended to alter the impairment profile such that slowing of response time was traded off for poorer accuracy. This tradeoff may be related partly to the fact that no feedback was given because without knowledge of response accuracy subjects will probably choose to optimise speed. Two caveats to this general explanation, however, need to be specified.

Firstly, in the category search task, the high lead group was both slower and less accurate with unrelated distractors. This implies less reliance on the fast holistic stage of categorisation postulated for the rapid rejection of nouns with a low relatedness to the category. ${ }^{27}$ With respect to practice, as the categories were familiar, subjects could gain implicit feedback by response monitoring and this would make them less susceptible to a general speedaccuracy tradeoff. Secondly, in the syntactic reasoning task, the high lead group was less cautious after practice and its improved reasoning times eliminate the initial slowness on complex statements. Syntactic reasoning is a typical controlled processing task where practice typically leads to more autonomic information processing modes ${ }^{1528} 30$ and the greater reliance on automatic processing might offset the initial lead deficits associated with complexity. Moreover as answers to the problems hinge on slight alterations in phrasing, subjects are likely to be less aware of their errors and, as a consequence, will have 
little incentive to slow down to avoid making them. It is worth making explicit that this implies that the low and medium lead groups were more able to monitor their errors as both groups improved their accuracy with practice.

These findings suggest that the issue of skill acquisition and the related transition between controlled and automatic information processing both need to be examined more specifically in workers exposed to lead, not least because they represent possible sources of cognitive difficulty. It is worth emphasising, however, that these are rather subtle alterations in performance and as such they are considerably less compelling and perhaps less important than the evidence for sensory motor slowing and impairment of memory. Indeed on the basis of the present results, with the exception of the mild central deficit noted earlier, a general increase in cognitive demands does not appear to differentially impair the performance of lead workers.

Previous neurobehavioural research on lead, and that reported here, have focused on relatively acute effects insofar as performance indices are assessed in relation to a measure of recent exposure; typically blood lead or ZPP concentrations. Indeed, it seems reasonable to suggest that some neurotoxic effects of lead are mediated by reversible neurochemical and membrane alterations that fluctuate directly in proportion to current lead concentrations. The possible link between neurochemical and psychological functioning, particularly the link between GABAergic function and response time, and dopaminergic function and attentional control has been noted before. ${ }^{6}$ The more compelling evidence for memory difficulties in the present study, albeit a circumscribed one, might suggest the involvement of the cholinergic system as this system had long been implicated in various memory disorders. ${ }^{31}$ As lead also disrupts this neurotransmitter system, it is conceivable that the areas of cognitive dysfunction identified here may be linked to the neurotransmitter systems known to be disrupted. ${ }^{32}$

As well as short term changes, it seems reasonable to propose that other neurotoxic effects of lead may be difficult to reverse because they arise from cumulative damage to neural structures. These effects, however, have proved more difficult to demonstrate and little evidence exists to show that cumulative exposure to lead determines the degree of cognitive impairment. For example, like many other studies, our present study found no evidence that the duration of lead exposure was correlated with impaired performance on any of the tasks. Part of the difficulty in demonstrating chronic effects can be traced to the problem of accurately determining a subject's exposure history and the further elucidation of chronic effects on the central nervous system will require an integrated measure of lead absorption. In recent years, the in vivo $x$ ray fluorescence of bone has emerged as a safe, accurate, and non-invasive method of estimating the accumulated body burden of lead, ${ }^{33-35}$ and the increasing use of this measure in studies of cognitive function is to be expected. As qualitative and quantitative changes in cognition are likely to occur with cumulative exposure, however, due attention needs to be given to this in assessments of the relation between cognitive function and chronic exposùre.

In conclusion, whereas it has been shown that lead can disrupt the performance of many psychological tasks, it has also been shown that the cognitive decline is not a generalised one. Some cognitive functions - such as sensory motor speed, attentional control, and certain aspects of memory-show clear declines whereas other memory and processing functions appear to be relatively well preserved. These results tend to strengthen the notion that lead disrupts a selected set of functions and highlights the importance of the development of models for neurotoxic impairment that attempt to specify the mental operations underlying the observed performance impairments. Such models need to make clear the parts played by sensory motor, attentional, and memory processes because these processes appear to be the most sensitive to exposure to lead. As such, our results suggest that studies of lead should move away from their previous reliance on clinically based tasks in their assessments, especially those that characterise performance in terms of single scores. Instead they should seek to investigate various aspects of cognition within the framework, methodologies, and models of modern cognitive psychology. ${ }^{36}$ Only by developing such models will it be possible to establish with confidence the particular set of cognitive processes responsible for alterations in performance after occupational exposure to lead at concentrations that are currently aceptable on purely medical grounds.

This work was supported by the Commission of European Communities and the Health and Safety Executive (UK). We thank the workers for their participation and the management, trade unions, and occupational physicians for assistance in coordinating the testing. We gratefully acknowledge the help of the factory occupational health departments for the collection of samples and the National Occupational Hygiene Service for the analysis of samples.

Requests for reprints to: Dr B T Stollery, Age and Cognitive Performance Research Centre, University of Manchester, Manchester M13 9PL.

1 Baker EL, Feldman RG, White RA, Harley JP, Niles CA, Dinse $\mathrm{GE}$, et al. Occupational lead neurotoxicity: a behavioural and 
electrophysiological evaluation. Study design and year one results. Br J Ind Med 1984;41:352-61.

2 Grandjean P, Arnvig E, Beckmann J. Psychological dysfunction in lead exposed workers: relation to biological parameters of exposure. Scand J Work Environ Health 1978;4:295-303.

3 Hanninen $H$, Hernberg S, Mantere P, Vasnato R, Jalkanen $M$. Psychological performance of subjects with low exposure to lead. J Occup Med 1978;20:283-9.

4 Valciukas JA, Lilis R, Eisinger J, Blumberg WE, Fishbein A, Selikoff IF. Behavioural indicators of lead neurotoxicity: results of a clinical field survey. Int Arch Occup Environ Health 1978;41:217-36.

5 Williamson AM, Teo RKC. Neurobehavioural effects of occupational exposure to lead. Br J Ind Med 1986;43:374-80.

6 Stollery BT, Banks HA, Broadbent DE, Lee WR. Cognitive functioning in lead workers. Br J Ind Med 1989;46:698-707.

7 Broadbent DE. Performance and its measurement. Br J Clin Pharmacol 1984;18:5S-9S.

8 Stollery BT. Measuring marginal toxicity in work environments. In: West R, Christie M, Weinman J, eds. Microcomputers, psychology and medicine. New York: J Wiley and Sons, 1990:97-115.

9 Mantree $\mathrm{P}$, Hanninen $\mathrm{H}$, Hernburg $S$. Subclinical neurotoxic lead effects: two-year follow-up studies with psychological test methods. Neurobehav Toxicol Teratol 1982;4:725-7.

10 Baker EL, White RF, Pothier LJ, Berkey CS, Dinse GE, Travers PH, et al. Occupational lead neurotoxicity: improvement in behavioural effects after reduction of exposure. Br J Ind Med 1985;42:507-16.

11 MacKay CJ, Cox T, Burrows G, Lazzwerini T. An inventory for the measurement of self-reported stress and arousal. $\mathrm{Br} \mathrm{J} \mathrm{Soc}$ Psychol 1979;17:283-4.

12 Karasek RA. Job demands, job decision latitude and mental strain: implications for job redesign. Administrative Science Quarterly 1979;24:285-308.

13 Jennrich J, Sampson P, Frane J. Analysis of variance with covariance including repeated measures. In: Dixon WJ, ed. BMDP statistical software. London: University of California Press, 1983:359-87.

14 Stollery BT, Broadbent DE, Lee WR, Keen RI, Healy TEJ, Beatty $P$. Mood and cognitive functions in anaesthetists working in actively scavenged operating theatres. $\mathrm{Br} J$ Anaesth 1988;61:446-55.

15 Hitch CJ, Baddeley AD. Verbal reasoning and working memory. Q J Exp Psychol 1976;28:603-21.

16 Maylor EA, Rabbitt PMA. Relationship between rate of preparation for, and processing of, an event requiring a choice response. $O J$ Exp Psychol $A$ 1989;41:47-62.

17 Wilkinson RT. Response-stimulus interval in choice serial RT: Interaction with sleep derivation, choice and practice. $Q J E x p$ Psychol A 990;42:401-23.

18 Seppalainen AM, Tola S, Hernberg S, Kock B. Subclinical neuropathy at "safe" levels of lead exposure. Arch Environ Health 1975;30:180-3.
19 Ashby JAS. A neurological and biochemical study of early lead poisoning. Br J Ind Med 1980;37:133-40.

20 Seppalainen AM, Hernberg S. Subclinical lead neuropathy. $\mathrm{Am}$ $J$ Ind Med 1980;1:413-20.

21 Araki S, Honma T. Relationships between lead absorption and peripheral nerve conduction velocities in lead workers. $\mathrm{Br} J$ Ind Med 1982;39:157-60.

22 Cavalleri A, Trimorchi F, Gelmi C. Effect of lead on the visual system of occupationally exposed subjects. Scand $J$ Work Environ Health 1982;8:suppl 1:148-51.

23 Criak FIM, Lockhart RS. Levels of processing: a framework for memory research. Journal of Verbal Learning and Verbal Behaviour 1973;12:599-607.

24 Eysenck MW. Incidental learning and orienting tasks. In: Puff $\mathrm{CR}$, ed. Handbook of research methods in human memory and cognition. 1982:197-228.

25 Brinley JF. Cognitive sets, speed and accuracy of performance in the elderly. In: Welford AT, Birren JE, eds. Behaviour, aging and the nervous system. Springfield IL: Thomas, 1965:119-49.

26 Cerella J. Information processing rates in the elderly. Psychol Bull 1985;98:67-83.

27 Smith EE, Shoben EJ, Rips LJ. Structure and process in semantic memory: a featural model for semantic decisions. Psychol Rev 1974;81:214-1.

28 Baddeley AD. Human memory: theory and practice. London Lawrence Erlbaum Associates, 1990

29 Shiffrin RM, Schneider $W$. Controlled and automatic human information processing: II Perceptual learning, automatic attending and a general theory. Psychol Rev 1977;84:127-90.

30 Hasher L, Zacks RT. Automatic and effortful processes in memory. $J$ Exp Psychol [Gen] 1979;108:356-88.

31 Kopelman MD. The cholinergic neurotransmitter system in human memory and dementia: a review. $Q J$ Exp Psychol $A$ 1986;38:535-73.

32 Silbergeld EK, Hruska RE. Neurochemical investigations of low level lead exposure. In: Needleman HK, ed. Low level lead exposure: the clinical implications of current research. 1980: 135-57.

33 Somervaille LJ, Chettle DR, Scott MC. In vivo measurement of lead in bone using x-ray fluorescence. Phys Med Biol 1985;30:929-43.

34 Scott MC, Chettle DR. In vivo elemental analysis in occupational medicine. Scand J Work Environ Health 1986;12:81-96.

35 Wedeen RD. Editorial: In vivo tibial XRF measurement of bone lead. Arch Environ Health 1990;45:69-71.

36 Stollery BT. Psychological toxicity: New methods for detecting subclinical effects. In: World Health Organisation. Environmental health 3: neurobehavioural methods in occupational and environmental health. Copenhagen: World Health Organisation, 1985:81-5.

Accepted 18 March 1991 